\title{
Research and application of closed cooling tower
}

\author{
Jianfeng Qian ${ }^{1, a}$, Lina $\mathrm{Li}^{1, \mathrm{~b}}$, Yankun Tan ${ }^{1, \mathrm{c}}$ and Dayu Zheng ${ }^{1, \mathrm{~d}}$ \\ ${ }^{1}$ School of Energy \& Civil Engineering, Harbin University of Commerce, Harbin, China \\ a qianjianfeng2002@163.com, ${ }^{b}$ lilina8902@126.com, ${ }^{c} 443200915 @ q q . c o m$, \\ dzhengdayu2000@126.com
}

Keywords: closed cooling tower, heat and mass transfer, optimal design

\begin{abstract}
This paper systematically reviews the research progress of the closed cooling tower at home and abroad. The abroad research of the closed cooling tower has been carried for a long time, so it is relatively mature. Although the domestic research has carried out relatively late, it also made a lot of achievements through the continuous efforts of the researchers for the study of the closed cooling tower. These studies to optimize the design of the closed cooling tower provide an important basis so that it can be applied to more areas.
\end{abstract}

\section{Introduction}

Because of these advantages of the closed cooling tower, it has a wide range of applications in the fields of electric power, metallurgy, chemical industry and building air conditioning. With more and more closed cooling tower applications, the study also received increasing attention. This paper systematically reviews the research of the closed cooling tower at home and abroad and application prospect.

\section{Theoretical Research of Abroad}

The abroad research of the closed cooling tower has been carried for a long time, it is relatively mature. Majumdar ${ }^{[1,2]}$ proposed heat and mass transfer control equation that based on the theory of two-phase. This is a successful numerical simulation, they also developed a two-dimensional commercial program, and it can be used for the design and calculation of a large cooling tower. Hypothesis the enthalpy of the saturated moist air and the temperature of water film between air and water film interface place in a linear relationship, and obtains a heat and mass transfer experience formula that the staggered tube bundle's external diameter is $19 \mathrm{~mm}$. The coefficient of convective heat transfer $h_{w}$ between tube outer deluge water and pipe outside surface is:

$$
h_{w}=704\left(1.39+0.022 t_{w}\right) G_{w}^{1 / 3} \text {. }
$$

Where $t_{w}$ is spray water film temperature, ${ }^{\circ} \mathrm{C}, G_{w}$ is deluge water mass flow, $\mathrm{kg} /\left(\mathrm{s} \cdot \mathrm{m}^{2}\right)$. Mass transfer coefficient $h_{d}$ between air and water film interface place is

$$
h_{d}=0.049 G_{a}^{0.905} \text {. }
$$

Where $G_{a}$ is air quality velocity, $\mathrm{kg} /\left(\mathrm{s} \cdot \mathrm{m}^{2}\right)$.

Cooling tower thermal characteristics of the theory study began in the 20th century $20 \mathrm{~s}$. The Equation of the Thermodynamic and differential pressure method and various experience formula is still used now. The method of Merkel Equation solution is improved. The Exchange number equation is solved by integration by Simpson is the easiest, but not perfect now. In especial, the closed-wet cooling tower is half empirical.

After closed cooling tower theoretical model and Heat and Mass Transfer Calculation experience formula is proposed, some researchers turn their focus into closed cooling tower optimization design and the research for heat and mass transfer enhancement. P. J. Erens ${ }^{[3]}$ found that adding plastic filler into the core could improve the heat transfer property of common circular tube cooler 
and it could instead of high price finned tube by comparing several different forms of evaporative condenser core body performance. Hasan and Siren ${ }^{[4]}$ compared the smooth circular closed cooling tower with finned circle closed cooling tower thermal performance to get a conclusion, Finned tube closed cooling tower of the heat and mass transfer coefficient is greater than the common round tube closed cooling tower; They also compared the smooth circular tube and oval tube closed cooling tower thermal performance in 2004, and got respectively suitable for the mass transfer experience formula through the data fitting, Oval tube closed cooling tower comprehensive performance coefficient is common round tube 1. 93-1.96 times ${ }^{[5]}$. Thacker ${ }^{[6]}$ designed a kind of closed cooling tower used in medium pressure nozzle, and test the spray density to provide the basis of closed cooling tower of the spray unit optimization design. Viljoen ${ }^{[7]}$ on the basis of Thacke: simulation closed cooling tower of spray water and air heat and mass transfer process, through the experiment test and CFD simulation to obtain the two kinds of low pressure, two kinds of medium pressure nozzle in air and calm state distribution characteristics (mainly including distribution uniformity degree, pressure drop and heat transfer characteristics).

\section{Theoretical Research of Domestic}

Although China carry out the research for the closed cooling tower a little late, the researchers is still working hard on it, and also got a lot of achievements.

Jin Feng ${ }^{[8]}$ presented a heat and mass Transfer mathematical model under three kinds of packing models. The parallel flat vertical channel along the liquid film in different high temperature distribution was studied with experiment and based on SIMPLE program on the basis of the numerical method. this paper put forward a new calculation model. Yang Qianglin made an improvement and modification on the basis of Merkel in the enthalpy difference method model, and presented a deep thermodynamic calculation on cross-flow evaporative cooler. And the influence of the various parameters on the cooling tower thermal performance was analyzed. Jiang Changjian etc. used for reference of WangTieJun's the crossflow cooling tower thermodynamic calculation mathematical model, and put the inside process water cross-flow evaporation cooling tower as the research object, this paper proposed a quasi three dimensional calculation method. Defines a series of dimensional for 1 parameter, through the calculated by the cross-flow $\varepsilon$ - NTU relationship diagram; discussed the various parameters on its thermal performance and the influence of $\varepsilon$-NTU relationship. Li Xueling ${ }^{[9]}$ studied the oval tube closed cooling tower on the theoretical and experimental, and analyzed the impact of elliptical tube closed cooling to the oval tube closed cooling tower water film heat and mass transfer relation. These studies put a theoretical and experimental basis for a new type of closed cooling tower optimization design and strengthen heat transfer.

\section{Conclusion}

Due to current foreign closed cooling tower price is much higher than domestic products, and domestic closed cooling tower is restricted to material performance and design and manufacturing level, and quality is not high, it can not reflect thermal efficiency advantages, comprehensive performance is not as good as foreign product, in a certain extent, these affected the application of the closed cooling tower in our country. Cloth water device and heat exchange coil (shown in Figure 1) is an important part of the closed cooling tower, through optimize and improve them can effectively improve the performance of closed cooling tower, so as to strengthen the outside of the tube heat and mass transfer efficiency.

From the above analysis, the closed cooling tower heat and mass transfer efficiency can be enhanced, there is a great space, and domestic theoretical research on closed cooling tower is not fully mature, the design is not reasonable and perfect. So, in order to improve the product quality of domestic and the development of energy efficient, it need to establish a theory system of closed cooling tower system and build system performance test platform, it has important significance to 
the market for the expansion of the closed cooling tower space and the development of low-carbon economy.

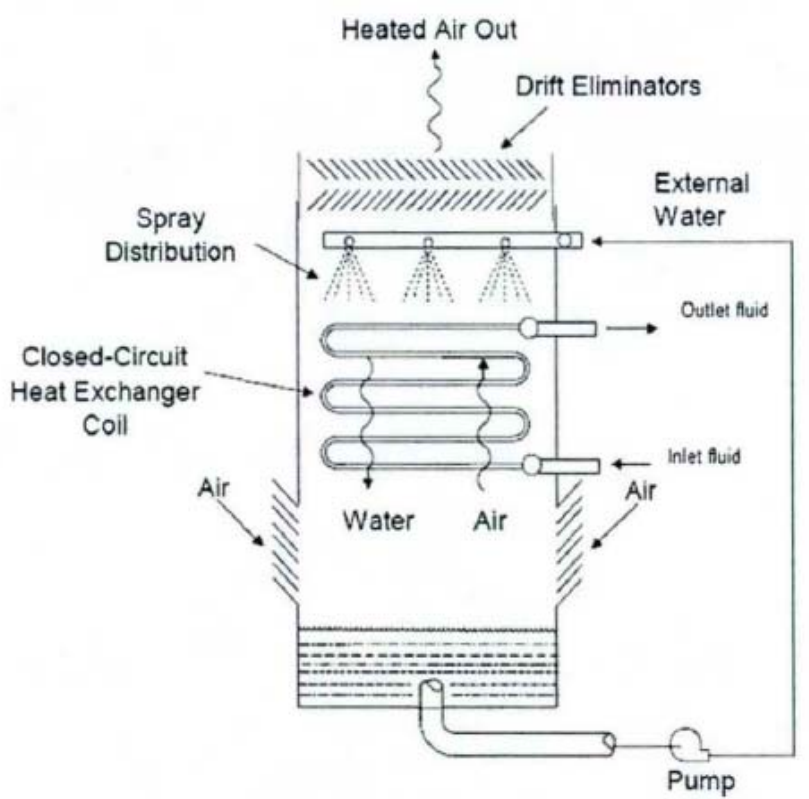

Fig.1 Closed cooling tower

\section{Acknowledgment}

The project has been sponsored completely by Project supported by the Natural Science Foundation of Heilongjiang Province (№. E201001).

\section{References}

[1] Majumdar A.K, Singhai A.K, Spalding D.B. Numerical Modeling of Wet Cooling Tower-Part1: Mathematical and Physical Models [J]. ASME Journal of Heat Transfer. 1983, 105:728-735.

[2] Majumdar A.K, Singhai A.K, Reilly H.E, Bartz J.A, Spalding D.B. Numerical Modeling of Wet Cooling Tower-Part2: Application to Natural and Mechanical Draft Towers [J]. ASME Joumal of Heat Transfer.1983,105:736-743.

[3] P.J. Erens. Comparison of some design choices for evaporative cooler cores [J]. Heat transfer engineering, 1988, (9):29-35.

[4] A. Hasan, K. Siren. Performance investigation of plain and finned tube evaporatively cooled heat exchangers [J]. Applied Thermal Engineering, 2003, 23(3):325-340.

[5] A. Hasan, K. Siren. Performance investigation of plain circular and oval tube evaporatively cooled heat exchangers [J]. Applied Thermal Engineering, 2004, 24(3):777-790.

[6] Thacker, J E. Design of medium pressure nozzles for cooling towers[D]. Mscing Thesis, University of Stellenbosch, 1997.

[7] D.J. Viljoen. Evaluation and performance prediction of cooling tower spray zones [D]. Mscing Thesis, University of Stellenbosch,2006.

[8] Lian zhiwei etc, Principles of heat and mass transfer and equipment [M],Beijing: China Construction Industry Press,2001.9:11-45

[9] Li xueling. The study of unsaturated evaporative cooler design and enhanced heat transfer properties [D]. Shanghai: East China University of Science and Technology Thesis, 2011 